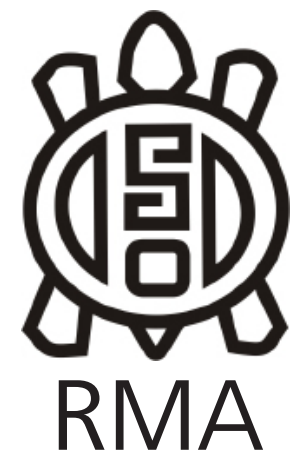

Dossier

\title{
Una aproximación a la alfarería de las comunidades aldeanas del final del periodo Formativo en Tilcara (Quebrada de Humahuaca)
}

\author{
An approach to the pottery of the village communities of the end of \\ the Formative period in Tilcara (Quebrada de Humahuaca)
}

Vanesa Beatriz Juarez*, Lucas Pereyra Domingorena**, Clarisa Otero***,
María Beatriz Cremonte****

*CONICET, Instituto de Datación y Arqueometría (CONICET- Provincia de Jujuy- UNT- UNJu). E-mail: vanesajuarezb@gmail.com

**CONICET, Instituto de las Culturas-UBA-CONICET. E-mail: lucasdomingorena@gmail.com

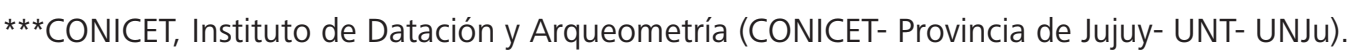
E-mail: clarisaotero@yahoo.com.ar

****CONICET, Instituto de Ecorregiones Andinas- UNJu-CONICET._E-mail: cremontebeatriz@gmail.com

\begin{abstract}
Resumen
La presente contribución apunta a visibilizar el estado actual de las investigaciones sobre el período Formativo de la localidad de Tilcara (Provincia de Jujuy), focalizándose en las prácticas alfareras, es decir, en los modos de hacer y consumir cerámica. Desde una perspectiva integradora, nuestro abordaje permitió comenzar a delinear nuevas caracterizaciones de aquellos recipientes considerados locales y propios de la época, como así también identificar casos de elaboración extrarregional. Los análisis a diferentes escalas (macroscópicos y microscópicos) efectuados sobre los diversos atributos de la alfarería contribuyeron al conocimiento de un repertorio tecnológico, morfológico y funcional poco estudiados. Así también, fue posible generar interpretaciones vinculadas con otros aspectos de las idiosincrasias de la población del sector central de la Quebrada de Humahuaca, tales como la trayectoria histórica de un particular modo de hacer la pasta cerámica y las formas de consumo durante el primer milenio D.C.
\end{abstract}

Palabras clave: Formativo; Tilcara; Alfarería; Rescates arqueológicos; Análisis integrador.

\begin{abstract}
This paper aims to visualize the current state of investigations centered in the Formative period of Tilcara (Jujuy province), focusing on pottery practices, that is to say, in the ways of doing ceramics. From a multi-dimensional perspective, our approach allowed us to start posing novel characterizations for those vessels considered locals and corresponding to that specific time frame, as well as identifying cases of extra-regional manufacturing. The analyses performed at different scales (macro and microscopic) over the different attributes of pottery, contributed to the knowledge of a technological, morphological, and functional repertoire scarcely understood. In this sense, it was also possible to generate interpretations linked to other aspects of the idiosyncrasies corresponding to the populations that inhabited the central sector of the Quebrada de Humahuaca, such as the historical trajectory of a particular way of doing ceramic pastes, and forms of consumption during the first millennia $A D$.
\end{abstract}

Keywords: Formative; Tilcara; Pottery; Archaeological rescue; Integrating analysis.

\section{Introducción y objetivos}

La Quebrada de Humahuaca es una de las áreas del Noroeste argentino que presenta mayor relevancia a nivel arqueológico porF la densidad de las investigaciones desarrolladas desde finales del siglo XIX. Sin embargo, a pesar de tal trayectoria, existen temas pendientes que carecen de información sólida para desarrollar, de manera completa, la historia ocupacional prehispánica de esta región, como es el caso de las sociedades aldeanas del primer milenio D.C. El área de estudio, el ejido urbano de Tilcara, cuenta con abundante información sobre el Pucará de Tilcara, sitio habitado entre los siglos XIII y XVI D.C. (Otero, 2013; Otero y Cremonte, 2010; Otero y Tarragó, 2017). No obstante, las ocupaciones previas al poblamiento de este gran conglomerado son escasas 
y se encuentran desdibujadas bajo los efectos erosivos de procesos naturales y antrópicos, pasados y presentes.

Durante los últimos treinta años, en el ejido urbano de Tilcara se detectaron más de una decena de contextos arqueológicos que arrojan evidencias sobre las primeras ocupaciones aldeanas definidas por otros autores como Formativo Final seguido por Humahuaca Temprano e Isla (Rivolta y Otero, 2016). En su mayoría se trata de sitios excavados por diversos investigadores, mediante técnicas de rescate luego de hallazgos accidentales (Otero y Mercolli, 2008; Otero y Rivolta, 2015; Rivolta, 1996; Rivolta y Albeck, 1992; Rivolta, Seldes y Mercolli, 2010).

En este trabajo, avanzamos en el estudio de la producción cerámica de las poblaciones aldeanas que habitaron la zona de Tilcara durante el período Formativo (siglos I a XI D.C.) (Otero y Rivolta, 2015). Centramos el análisis en los últimos siglos de este rango cronológico dado la evidencia con la que contamos. Para tal fin, se examinaron los conjuntos alfareros registrados en la intersección de las calles Lavalle y Sorpresa (Til.22) y el hallado en el domicilio de la familia Carrazana Paredes en el barrio Malka (Malka-CP) ( Figura 1), cuyas ocupaciones se ubican cronológicamente entre los siglos VII y XI. El análisis de estos conjuntos junto a la revisión de la documentación registrada por los investigadores que realizaron los rescates, fue crucial para integrar la información generada a nivel funcional y contextual. Así, se buscó articular las evidencias para avanzar en una primera sistematización del material cerámico de finales del Período Formativo de Tilcara.

La alfarería de este período fue descripta a partir de múltiples hallazgos de grandes ollas tubulares y tazones, del Tipo Negro sobre rojo. Este componente cerámico, en referencia a la bicromía de la representación en pintura negra sobre el fondo rojo, ha sido interpretado, como el puntapié inicial para una tradición regional alfarera conocida como Omaguaca (Menacho y González, 2005; Nielsen, 2007; Otero y Rivolta, 2015). Los atributos

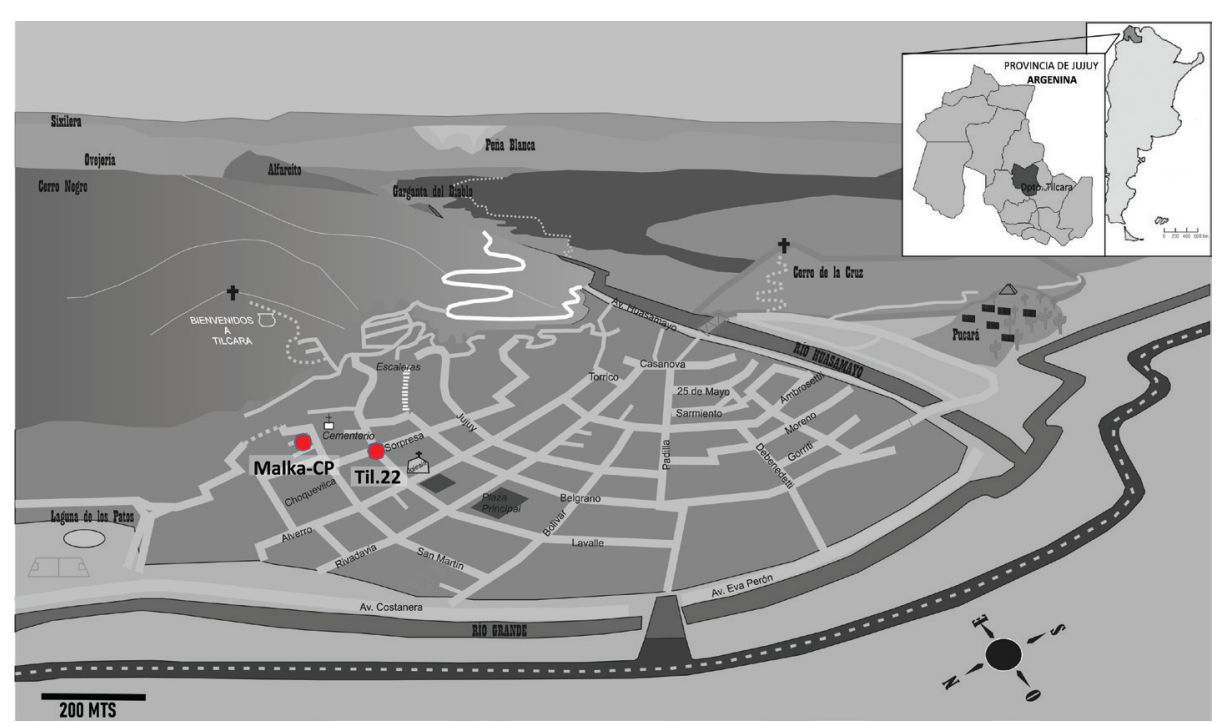

ya conocidos fueron enriqueciéndose a partir de la identificación de nuevas formas, recursos plásticos y técnicas de manufactura que hemos detectado en los últimos años. De allí que nuestra propuesta sea continuar con la caracterización estilística de la cerámica para lograr precisar aún más los elementos brevemente descriptos en los informes consultados, y complejizar el conocimiento de los hallazgos y sus contextos arqueológicos descubiertos de manera fortuita. Así también, la abundancia y variabilidad de los materiales cerámicos permitieron avanzar en una caracterización tecnológica desde la petrografía de sus pastas. De esta forma entonces, se comienza a inferir los modos de hacer alfarería que, a su vez, revelarían los rasgos propios de las idiosincrasias locales y la posible demarcación de 'fronteras' o distancias sociales con otros grupos que habrían mantenido vínculos con las poblaciones de la Quebrada de Humahuaca a lo largo de su historia prehispánica (Cremonte, 2006; Rivolta, Greco y Otero, 2017). Por otro lado, se trabajó en la determinación de los usos que pudieron haber tenido cada uno de los recipientes analizados, revelando comportamientos de consumo y almacenaje, distintos a los que se distinguen para períodos posteriores.

Además de los conjuntos aquí estudiados, el registro del período Formativo se conoce mediante hallazgos registrados en otros domicilios de la planta urbana de Tilcara: Familia Mulqui (Til.20) (Mendonça, Bordach, Ruiz, Cremonte, 1991); Familia Arroyo (Til.23) (Madrazo, 1968); Familia Flores (Mercolli, Zaburlín y Seldes, 2004); Hotel Intiwayna (Rivolta et al., 2010); Hotel El Antigal (Madrazo, 1968; Otero y Rivolta, 2015); y otros contextos fortuitos en el barrio Malka (Nielsen, 2001). Estos materiales se encuentran en guarda en el Museo Arqueológico "Dr. Eduardo Casanova" del Instituto Interdisciplinario Tilcara, FFyL-UBA, donde fueron consultados.

\section{Los sitios arqueológicos del Período Formativo en Tilcara}

Los conjuntos cerámicos presentados en este trabajo corresponden a materiales recuperados en los sitios Til.22 (Rivolta y Albeck, 1992) y Malka-CP (Valda, 2010). Ambos, se conocen

Figura 1. Localización de la esquina Lavalle y Sorpresa (Til.22) y domicilio de la familia Carrazana Paredes (Malka-CP) en la planta urbana de Tilcara.

Figure 1. Location of the corner Lavalle and Sorpresa (Til.22) and residence of the CarrazanaParedes family (Malka-CP) in the urban plant of Tilcara. 
a raíz de trabajos de rescates que se realizaron en marzo de 1990 y agosto de 2010, respectivamente. Los asentamientos Til.22 y Malka-CP fueron identificados como espacios habitacionales del primer milenio D.C., contando el primer caso con dataciones radiocarbónicas y el segundo con asociaciones contextuales.

El sitio Til.22 corresponde a un hallazgo detectado en superficie luego de la nivelación de una calle y una posterior tormenta de granizo que terminó dejando expuesta una importante cantidad de materiales depositados sobre un muro ( Figura 2.a). La concentración superficial fue registrada por Rivolta y Albeck (1992) como un basurero, mientras que el muro se identificó como parte de una vivienda sepultada por un área de descarte, posiblemente de una posterior ocupación. Los fechados de tres muestras de carbón recuperadas en el basurero arrojaron una antigüedad de $940 \pm 60$ AP (LP 336); $1025 \pm 140$ (LP 349) y $1160 \pm 80$ (B 80704) (Rivolta, 1996, p. 132; Tarragó y Albeck, 1997). El registro estaba compuesto además por restos óseos de fauna, puntas de proyectil pedunculadas de obsidiana y sílice, y objetos cerámicos muy fragmentados, preponderando recipientes sin representaciones plásticas, junto a dos modelados zoomorfos de cerámica ( Figura 2.d y e) en muy buen estado de conservación (Rivolta, 1996). El conjunto alfarero registrado contiene tiestos que corresponden a piezas denominadas ollas tubulares ( Figura 2.f), tazas y tazones con representaciones geométricas ( Figura 2.b y c), previamente identificadas como propias de una producción local. Además, se reconocieron otros elementos que permiten postular la existencia de interacción social con poblaciones que habitaron paisajes transversales a la Quebrada de Humahuaca. Esto se evidencia por la presencia de fragmentos San Pedro Negro Pulido (Figura 2.g) que, según su petrografía, correspondería a una producción local en los oasis de Atacama, Norte de Chile (Rivolta y Otero, 2015; Otero y Rivolta, 2016). Así también, se registró la presencia de un caparazón del molusco Stropocheilus oblongus lorentzianus, característico de los valles húmedos, posiblemente procedente de las tierras bajas orientales de la provincia de Jujuy (Rivolta, 1996).

Por otro lado, Malka-CP es un sitio emplazado en el barrio Malka, hallado durante el trabajo de rescate efectuado durante la ampliación de una vivienda en el domicilio de la familia Carrazana Paredes. Las tareas de salvataje estuvieron a cargo del personal del Instituto Interdisciplinario Tilcara quienes registraron ocho ollas tubulares semicompletas (Figura 3.a). Junto a estas ollas se encontraron recipientes cerámicos de menor tamaño (Figura 3.d, e, f, h e i), una elevada cantidad de tiestos, una pieza cerámica zoomorfa fragmentada ( Figura 3.g), numerosas cuentas de collar (Figura 3.b), artefactos de metal, entre ellas una vincha de oro (Figura 3.c), un fogón, y cuatro estructuras funerarias (tres en cámaras sepulcrales y un entierro directo) (Otero y Rivolta, 2015; Valda, 2010). Si bien Malka-CP no posee fechados publicados, se tiene en cuenta a modo de referencia los

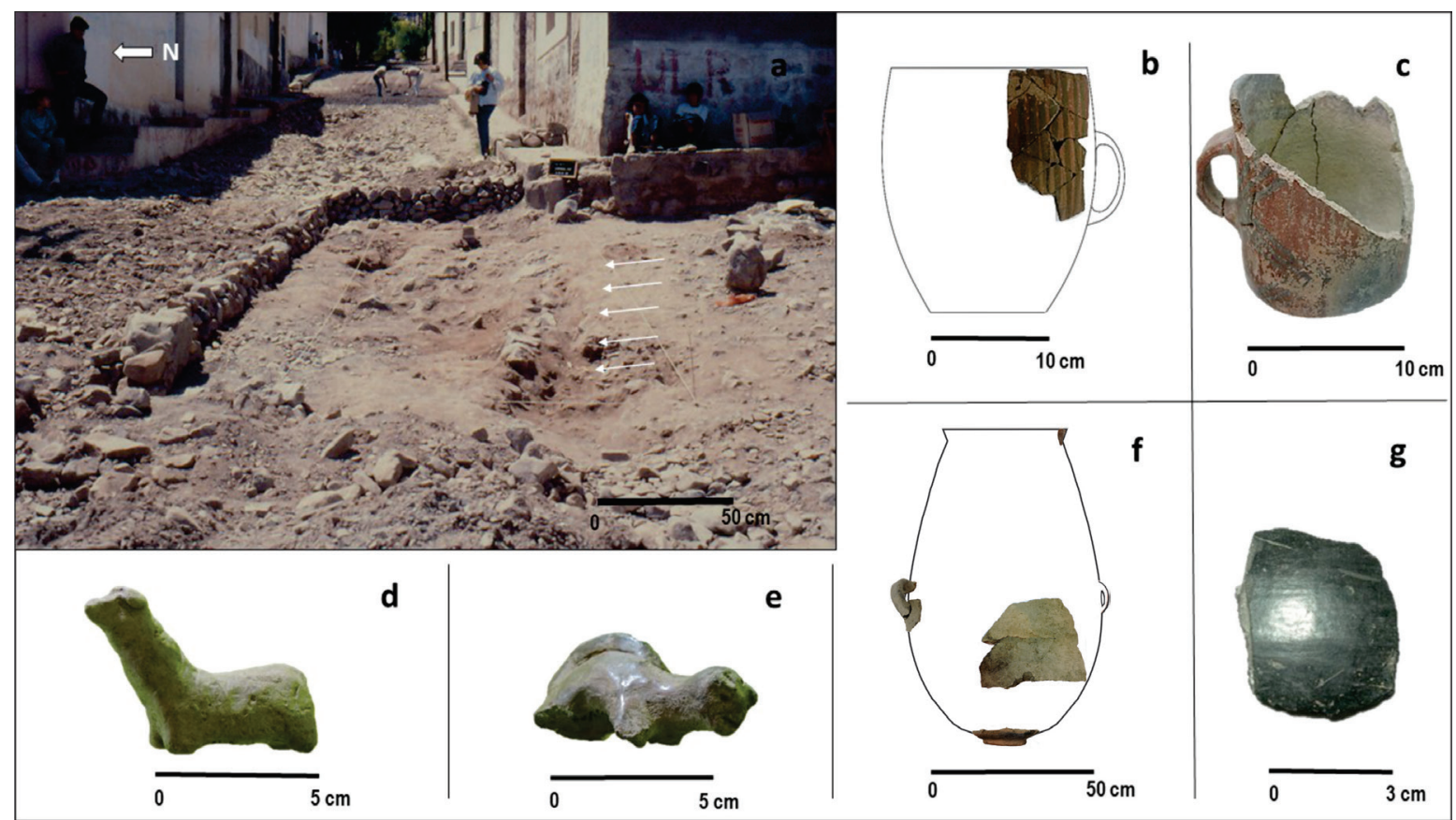

Figura 2. Hallazgos en Til.22. a. Muro sepultado (esquina Lavalle y Sorpresa); b. Contorno de tazón subcilíndrico; c. Taza de paredes rectas; d-e. Modelados zoomorfos; f. Reconstrucción hipotética de olla tubular; g. Tiesto San Pedro Negro Pulido.

Figure 2. Findings in Til.22. a) buried wall (Lavalle and Sorpresa corner); b. Shape of a sub-cylindrical big cup with handle (tazón); $c$. Straight-walled cup; d-e. Zoomorphic mouldings; f. Hypothetical reconstruction of a tubular pot; $g$. Black Polished San Pedro ceramic fragment. 


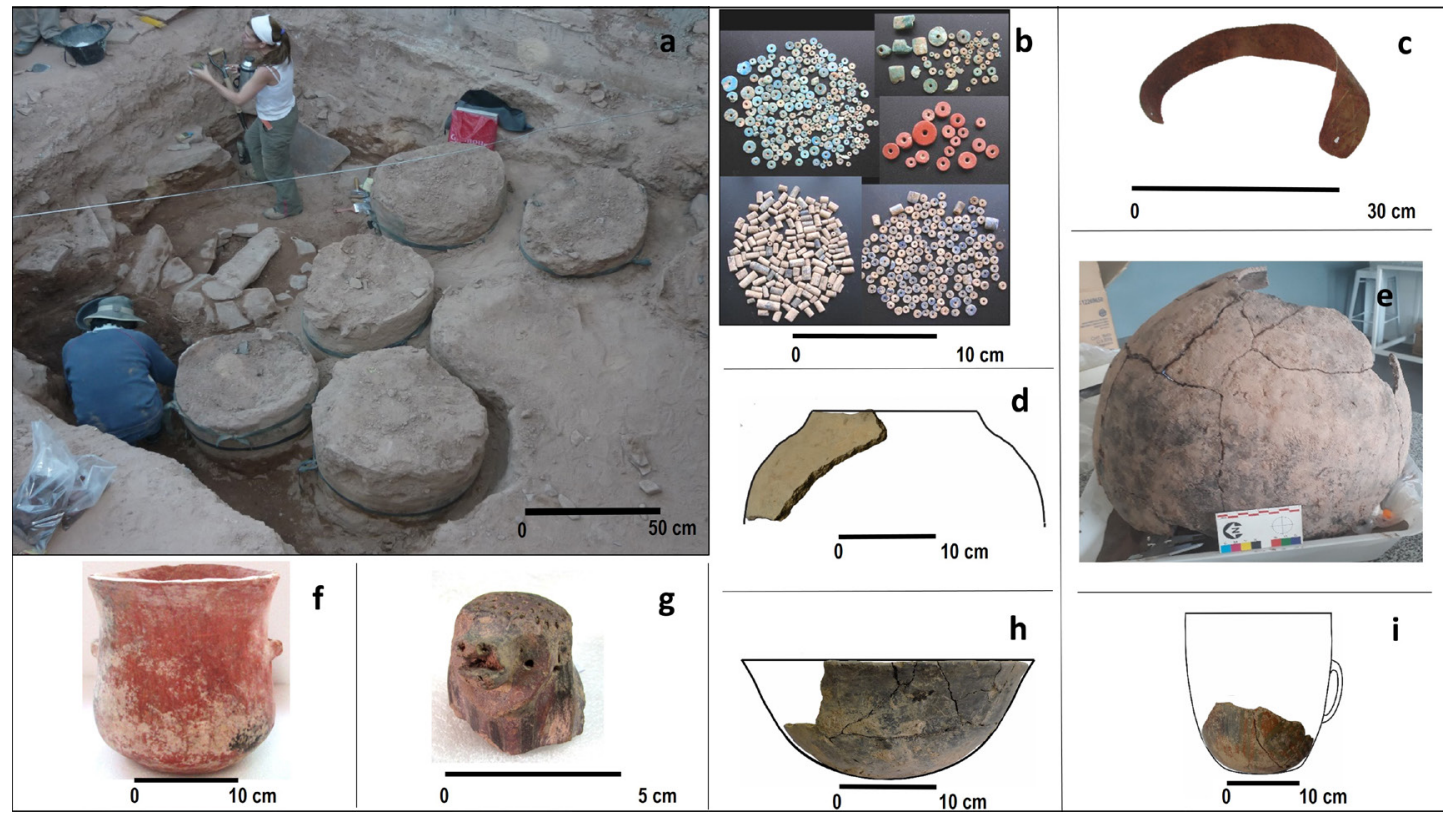

Figura 3. Hallazgos en Malka-CP. a. Conjunto de ocho ollas tubulares in situ (Fotografía cortesía Noel Montoya. Centro Universitario Tilcara - UBA); b. Cuentas de collar; c. Vincha de oro; d. Contorno de olla; e. Olla globular; f. Vasija restringida; g. Fragmento de pieza zoomorfa; h. Escudilla; i. Contorno de tazón subcilíndrico.

Figure 3. Findings in Malka-CP. a. Assemblaje of eight tubular pots in situ (Photography cortesy of Noel Montoya. Centro Universitario Tilcara - UBA); b. Collar-beads; c. Gold headband; $d$. Shape of pot; e. Globular pot; f. Restricted vessel; $g$. Fragment of zoomorphic piece; $h$. Shallow bowl; i. Shape of sub-cylindrical big cup with handle (tazón).

hallazgos trabajados anteriormente por Nielsen (2001), quién durante la construcción de viviendas emplazadas en el mismo barrio, enmarcadas en un proyecto del gobierno provincial, registró contextos con materiales equiparables, datados en 990 \pm 50 AP (LP-988).

\section{Estrategias y metodologías}

Las piezas estudiadas resultaron de la determinación de un Número Mínimo de Vasijas (NMV), a partir de la identificación de siluetas de morfologías específicas, y de familias de fragmentos (Orton, Tyers y Vince, 1997), cuando los tiestos se reconocieran como partes de una misma pieza y compartieran atributos como espesor, cocción, tratamiento de superficie y color. Para corroborar las morfologías y dimensiones inferidas en los recipientes, éstos fueron comparadas con objetos recuperados en otros sitios contemporáneos de Tilcara y que, actualmente forman parte de las colecciones salvaguardadas en el Museo Arqueológico "Dr. Eduardo Casanova" de Tilcara.

Con respecto a las muestras recuperadas en Malka y Til 22 se analizaron 23 piezas semicompletas y 1949 tiestos procedentes de ambos sitios. El repertorio morfológico propuesto se generó a partir del análisis tanto de los recipientes semicompletos como de los grupos de fragmentos que permitieron su remontaje para el reconocimiento de siluetas. La diversidad morfológica de las vasijas se registró cualitativamente, organizando la muestra en categorías que son propias de este tipo de contextos arqueológicos, algunas previamente definidas y otras recientemente identificadas. Para su clasificación se consideraron nomenclaturas registradas en la bibliografía con el fin de homogeneizar el léxico morfológico (Balfet, Fauvet-Berthelot y Monzón, 1983; Cremonte y Bugliani, 2006-2009; Ortiz y Delgado, 1997). Asimismo, el progresivo y exhaustivo registro de atributos como forma, volumen, grado de estabilidad, transportabilidad, tratamiento de superficie y recursos plásticos, en asociación a rastros de desgastes y adherencias, brindaron información acerca de posibles funciones y/o usos vinculados a cada caso (Menacho, 2007; Rice, 1987; Skibo, 1992).

Finalmente, algunos atributos técnicos de la alfarería -tanto la proporción arcilla-antiplástico como el tamaño de los mismos- se abordaron mediante análisis petrográficos de las pastas cerámicas. Para ello, se utilizó un microscopio de polarización Leica DM750P donde se caracterizaron 25 secciones delgadas, asociadas al correlato estilístico de la alfarería, considerándose rasgos cualitativos y cuantitativos de los componentes de dichas pastas. Las fases plásticas y los elementos no plásticos menores a 15 micrones fueron incluidos como parte de la matriz arcillosa teniéndose en cuenta coloración y estructura (Curtois, 1976). Posteriormente se determinó la mineralogía de las inclusiones mayores a 15 micrones distinguiendo principalmente entre cristaloclastos y 
litoclastos, como así también tiesto molido, nódulos de arcilla y opacos. Se analizó la distribución modal de los componentes de las pastas (point counting) (Pereyra Domingorena y Cremonte, 2017). Los datos obtenidos se relacionaron con la geología local (González M., Tchilinguarian, Pereyra, Ramallo y González O., 2003) para plantear hipótesis sobre los posibles lugares de producción y sobre obtención de materias primas.

\section{Resultados obtenidos}

En primera instancia, en el conjunto cerámico estudiado fue posible discernir variabilidad tipológica, identificándose grupos de fragmentos y/o piezas de los tipos: Ordinario; Ordinario deleznable; Gris alisado; Gris amarronado; Gris pulido; Gris negro pulido; Gris inciso pulido; Naranja claro pulido; Marrón claro pulido; Rojo pulido y Negro sobre rojo. En general, cada tipo fue asociado a un correlato morfológico. En el caso de los fragmentos Gris inciso pulido no fue posible establecer una correlación morfológica específica.

Las formas relevadas en las muestras de ambos sitios, para este período, se caracterizan por la presencia de

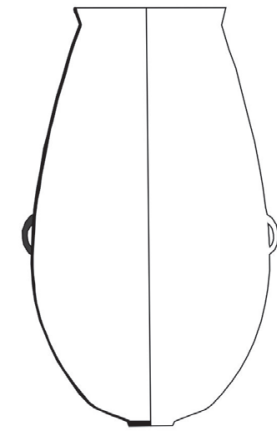

a
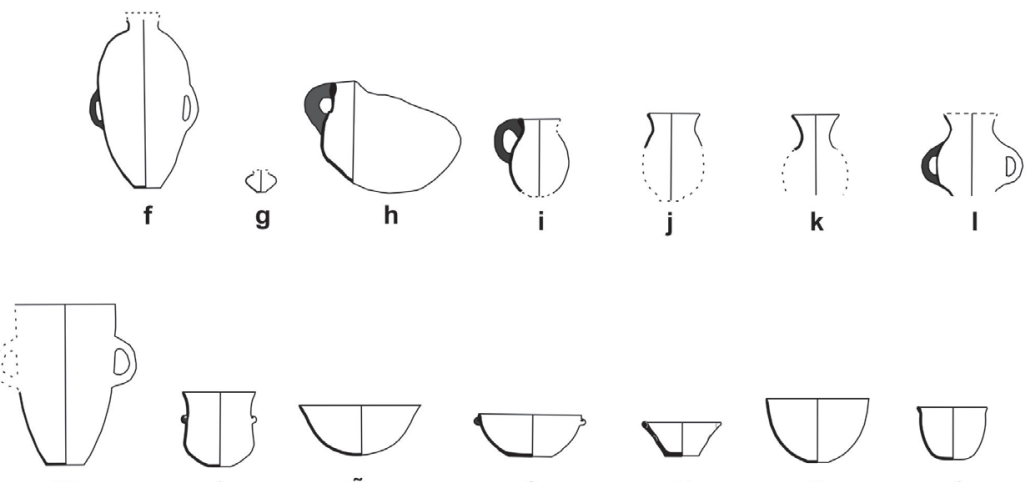

m
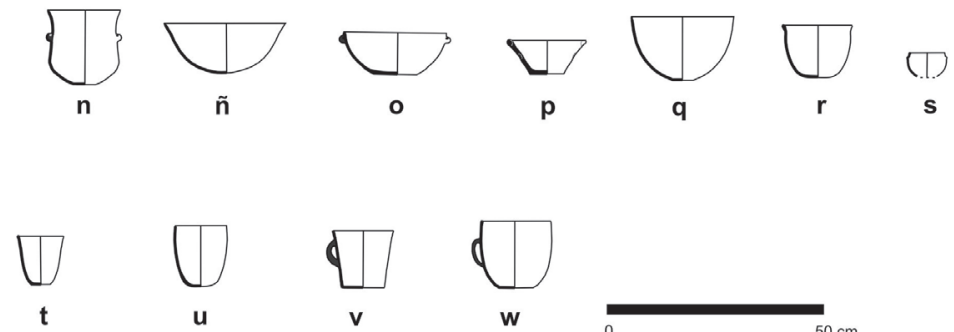

Figura 4. Repertorio morfológico de Malka CP y Til.22. a. Olla tubular con base plana; b. Olla tubular con base cóncava; c. Olla subglobular; d. Olla de cuello cilíndrico largo; e. Olla globular de cuello incipiente; f. Cántaro; g. Cántaro pequeño; h. Botella calceiforme; i. Jarra ovoide; j. Tinaja ovoidal; k. Tinaja subglobular; I. Tinaja subglobular con asas; m. Balde de contorno simple; $n$. Balde restringido; ñ. Escudilla semiesférica de contorno ligeramente inflexionado; o. Escudilla semiesférica de contorno simple con asas; p. Escudilla troncocónica con asas; q. Cuenco semiesférico de contorno simple; r. Cuenco subglobular de borde divergente; s. Cuenco subglobular con apéndice mamelonar; t. Vaso de borde divergente; u. Vaso subcilíndrico; v. Taza troncocónica; w. Tazón subcilíndrico.

Figure 4. Morphological repertoire of Malka CP and Til.22 sites. a. Tubular pot with plane base; $b$. Tubular pot with concave base; c. Sub-globular pot; d. Pot with long cylindrical neck; e. Globular pot with incipient neck; f. Pitcher; g. Small pitcher; $h$. Calceiform bottle; i. Ovoid jug; j. Oval jar (tinaja); k. Sub-globular jar (tinaja); I. Sub-globular jar with handle (tinaja); $m$. Simpleprofile big beacker (balde); n. Big beacker (balde); ñ. Shallow bowl; o. Shallow bowl with handle; p. Frustoconical shallow bolw with handle; q. Bowl; r. Sub-globular bowl; s. Sub-globular bowl with modelling appliqué; t. beacker; u. Sub-cylindrical big cup; v. Frustoconical cup; w. Sub-cylindrical big cup with handle (tazón). 
grandes ollas tubulares que alcanzaron el metro de altura (Figura 4.a y b), tazas y tazones, llamados usualmente vasos en la bibliografía (Figura 4.v y w). Algunos de éstos fueron también registrados en sitios como Estancia Grande (Olivera y Palma, 1997), Calaverioj (Zaburlin, 2009) y Alfarcito (Madrazo, 1969) en quebrada y puna de la provincia de Jujuy. Estos tipos de recipientes, que ya eran conocidos, están muy presentes en Tilcara y particularmente en Til.22 y Malka- $C P$, donde además se identificaron nuevas morfologías como ollas de menores dimensiones (Figura 4.c, d y e), cántaros (Figura 4.f y g), botellas calceiformes (Figura 4.h); jarras (Figura 4.i), tinajas (Figura 4.j, k y l), baldes (Figura 4.m y n), escudillas (Figura 4.ñ, o y p), cuencos (Figura 4.q, r y s) y vasos (Figura 4.t y u) que se presentan, mayormente, sin representaciones plásticas. Las únicas excepciones a este patrón son las tazas (Figura 4.v) y tazones (Figura 4.w) con diseños de líneas paralelas y/o quebradizas en pintura negra sobre fondo rojo y un fragmento de recipiente cuyo borde presenta una impresión rítmica de dedos arrastrados.

La funcionalidad de los recipientes identificados pudo inferirse considerando morfologías, volúmenes, estabilidad, transportabilidad, tratamientos de superficie, huellas de uso y contextos de asociación de los conjuntos cerámicos. En principio, la variabilidad de tamaños registrados indicaría tanto diferentes funciones como distintas formas de consumo.

Las características específicas de los grandes contenedores cerámicos, como las ollas tubulares (Figura 4.a y b), que presentan una capacidad volumétrica hasta 200 litros, alturas de poco más de un metro y paredes de un grosor fino en relación con las dimensiones de los recipientes, sugieren que fueron piezas de difícil transporte y que tuvieron un uso comunitario. Asimismo, la ausencia de desgaste interno y de rastros de hollín en sus superficies indicaría que fueron usados principalmente para la conservación de alimentos u otros recursos, más que para su procesamiento. El contexto de hallazgo de Malka-CP afianzaría esta interpretación sobre la comensalidad colectiva y la función de almacenaje para estas piezas. Las ocho ollas tubulares halladas estaban en un mismo nivel (las bases se encontraron por debajo del piso de ocupación, lo cual se interpreta como una disposición fija que habría dejado al descubierto dos terceras partes de las ollas), poseen similares capacidades de volumen y no presentaban rastros de exposición al fuego o desgates que pudieran resultar de actividades como raspados internos que suelen producirse al revolver la comida durante la cocción. Además, una de ella contenía un tazón (Figura 4.w) en su interior, por lo cual pensamos que habría servido como recipiente para extraer una parte fraccionada del abundante contenido almacenado, quizá granos.

En cambio, las ollas de tipo globulares y subglobulares (Figura 4.c, d y e), que no superan los $60 \mathrm{~cm}$ de alto, presentan formas y dimensiones aptas para la conservación de calor. Además, por el tipo de tratamiento de superficie que poseen se puede considerar que respondieron satisfactoriamente al shock térmico por exposición al fuego. La mayaría de las superficies externas de estos recipientes, sin tratamientos plásticos, poseen cierta rugosidad, lo que según Rye (1981) daría una mayor resistencia a las paredes. Asimismo, estas piezas están impregnadas de hollín, observándose colores claroscuros correspondiente al recocido de la vasija. Estos indicadores sugieren que estos recipientes estuvieron vinculados al procesamiento de alimentos, que pudieron ser el hervido, el tostado o el guisado. La excepción a este patrón corresponde a una olla globular de cuello corto (Figura 4.e), que se distingue por presentar un pulido externo muy logrado, lo cual sugiere que habría sido utilizada para el servicio o el almacenaje.

Tanto los cántaros, que en ningún caso supera los 60 $\mathrm{cm}$ de altura (Figura 4.f), como las botellas calceiformes (Figura 4.h) pudieron ser piezas aprovechadas para el almacenaje y traslado de líquidos a pequeñas y medianas distancias, siendo que se trata de vasijas restringidas que por su volumen no contendrían recursos pesados de transportar. Por otro lado, las superficies externas pulidas de algunos cántaros demuestran la intención de reducir la permeabilidad de los recipientes, particularmente líquidos. Las jarras y tinajas (Figura 4.i, j, k y l), de forma semejante, por su pulido debieron permitir el almacenamiento y también el servicio de líquidos, a menor escala dadas sus dimensiones más pequeñas.

Con relación a los baldes (Figura 4.m y n) se trata de recipientes abiertos y medianos, que por el tipo de contorno y silueta no favorecerían la retención del calor. Además, presentan superficies con engobes o pinturas y pulido en ambas caras, que demuestran la intención de exhibir estas piezas durante su uso, quizás como recipientes para el servicio. Estos atributos se suman a la ausencia de rastros de desgaste y adherencias de residuos carbonizados u hollín, por lo que es válido estimar que su uso habría estado más bien relacionado con mostrar los alimentos contenidos.

Los tamaños de las escudillas (Figura 4.ñ y o), cuencos (Figura 4.q y r), y tazones (Figura 4.w) sugieren piezas que podrían haber sido transportadas a cortas y largas distancias e ideales como vajilla de servicio. Particularmente, el diámetro de apertura presente en cuencos y escudillas posibilita tanto el servicio como la exposición de alimentos o recursos conservados en su interior. Sin embargo, una escudilla registrada en MalkaCP con hollín en la superficie externa evidencia que la pieza fue expuesta al fuego, posiblemente para calentar alimentos.

Los vasos subcilíndricos de bordes divergentes (Figura 4.t y u), tazas (Figura 4.v) y tazones constituyen piezas que 
presentan una capacidad de volumen que varía entre 0,250 litros y 1 litro aproximadamente. La superficie externa de estas piezas presenta usualmente diseños geométricos en color negro sobre fondo rojo seguido de un pulido. La presencia de pintura y pulido puede extenderse hasta el sector interno del borde del recipiente. Las dimensiones, las morfologías y los tratamientos mencionados sugieren un uso de estos recipientes como vajilla de servicio, posiblemente limitados al consumo individual de líquidos o alimentos semiespesos.

Las piezas diminutas, con formas a modo de cántaros, escudillas y cuencos, halladas en Malka-CP (Figura 4.g, p y s), presentan características morfológicas que permitan asociarlas al consumo de comidas y bebidas. Sin embargo, sus pequeñas dimensiones indicarían funciones ajenas al servicio de alimentos en contextos domésticos. En este sentido, se interpreta a estas peculiares piezas como ofrendas mortuorias considerando que objetos como éstos fueron registrados en tumbas de finales del primer milenio D.C en el Desierto de Atacama (Uribe, 2002), un área con quienes los habitantes del Formativo Final de Tilcara tuvieron vínculos (Menacho y González, 2005; Otero y Rivolta, 2015; Rivolta y Albeck, 1992; Tarragó, 1977). Por su parte, las figurinas zoomorfas de Til.22 se interpretan como "representaciones de..." en la rogativa por la abundancia, como illas en donde su utilización se vincula más con lo que significan o representan (Otero, 2013; Pimentel, 2009).

El análisis petrográfico se efectuó sobre 25 muestras extraídas de los diversos tipos cerámicos. Se examinaron fragmentos representativos de piezas de los tipos: Ordinario (ollas, jarras y tinajas); Ordinario deleznable (ollas globulares); Gris alisado (escudillas); Gris amarronado (ollas); Gris pulido (escudillas, vasos); Gris negro pulido (tinajas, cántaros); Gris inciso pulido (sin formas identificadas); Naranja claro pulido (baldes); Marrón claro pulido (olla globular); Rojo pulido (piezas abiertas: vasos o cuencos); y Negro sobre rojo (tazas y tazones).

El estudio comprobó distintos procedimientos de manufactura. En primer lugar, el más representado (92\% de las muestras $n: 23$ ) se asocia al agregado de litoclastos de pizarra/filita, que provendrían de la Formación Puncoviscana (González et al., 2003), en la cual están asentados los sitios donde se hallaron los tiestos estudiados. Entonces, por la correspondencia entre estos elementos no plásticos y la geología local, se infiere el carácter local de la producción alfarera de la mayoría de los tipos cerámicos analizados. La incorporación intencional de estas rocas metamórficas de bajo grado (pizarra/filita) dentro de la pasta cerámica constituye también un rasgo de identidad de la producción alfarera del Período Tardío e Inca, en la Quebrada de Humahuaca (Larcher, Otero y Cremonte, 2016).

Por otra parte, la composición de las dos vasijas restantes
(Marrón claro pulido y Gris pulido inciso) evidenció la presencia de componentes ajenos al escenario geológico donde se emplazan nuestros casos de estudio. En estas dos muestras se registró arcillita, arenisca y tiesto molido, lo cual trazaría interacciones con la vertiente oriental de la Quebrada de Humahuaca. Por ejemplo, en el fragmento inciso analizado, que presentó en su composición tiesto molido en porcentaje y granulometría semejante al denominado Grupo 2 de la Tradición San Francisco (Pereyra Domingorena y Cremonte, 2017), se combina tanto la pasta cerámica como la representación plástica incisa de dicha tradición alfarera de las yungas jujeñas.

\section{Discusiones y conclusiones}

Los resultados obtenidos en este trabajo ampliaron, desde una perspectiva integradora, el repertorio estilístico escasamente conocido para las ocupaciones aldeanas correspondientes a los últimos siglos del primer milenio D.C., detectadas hasta el momento en la planta urbana de Tilcara. Esto posibilitó avanzar en las propuestas sobre los modos de hacer alfarería para luego integrar tales interpretaciones a las aproximaciones sobre sus posibles usos.

Así, por ejemplo, la presencia de recipientes de gran tamaño, como las ollas tubulares especialmente concentradas en Malka-CP, podría manifestar una forma de consumo que involucró un gran número de actores, dado su agrupamiento y alta capacidad de almacenaje. La abundancia de recipientes para el servicio -cuencos, escudillas y tazones- sugiere la participación de éstos en eventos de consumo comunal, donde las dimensiones de las piezas habrían sido aptas para contener porciones de alimentos que excedían una ración individual. Asimismo, los acabados se superficie, particularmente los pulidos muy logrados y las expresiones plásticas del Tipo Negro sobre rojo podrían considerarse como atributos ligados a su función pública, es decir, como trasmisores de mensajes iconográficos que pudieron haber contribuido a la conformación de una identidad Omaguaca (Otero, 2017). Sin embargo, es importante destacar que las ollas tubulares de gran tamaño no figuran en el registro arqueológico de los períodos posteriores, es decir, serían piezas exclusivas de la etapa final del Formativo, indicando una discontinuidad entre las prácticas de consumo de las sociedades aldeanas de finales del primer milenio D.C. y los habitantes de los poblados más conglomerados del Período Tardío en este sector de la Quebrada de Humahuaca.

Un aspecto interesante que resalta esta investigación reside en las funciones atribuibles a los recipientes muy pequeños registrados en Malka-CP, pues consideramos que estos objetos en su carácter de miniaturas, y sin evidencias de rastros de uso (desgastes y/o adherencias), constituyeron parte de ofrendas mortuorias como sucede en sitios contemporáneos de la puna chilena. Por su parte, 
la presencia de representaciones zoomorfas en Til.22 da cuenta también de la vigencia de prácticas ceremoniales en donde estas figuras en miniatura tienen una activa participación como ofrendas en enterratorios (Núñez, Cartajena, Carrasco, De Souza y Grosjean, 2006), y/o illas, en espacios productivos (Carlos Ríos, 2015).

El abordaje tecnológico desde la petrografía nos aproximó al conocimiento de las maneras de hacer cerámica asociadas a diferentes variedades estilísticas, reconociéndose además en los resultados indicios sobre la producción local y alóctona. En la gran mayoría de los casos estudiados, determinamos una base técnica homogénea con continuidad en el período posterior vinculado a lo que se denominó manufactura Omaguaca (Nielsen, 2001) en la Quebrada de Humahuaca, es decir, una posible marca de identidad social en la región (Larcher et al., 2016), que perduró durante casi un milenio (entre los siglos VII y XVI D.C.). Por otro lado, la caracterización petrográfica de las vasijas permitió evidenciar parte de las relaciones extrarregionales de este sector de las aldeas tilcareñas del Período Formativo. Los resultados obtenidos mostraron vínculos orientados al contacto con poblaciones de las tierras bajas orientales de la provincia de Jujuy. Este trabajo, suma un nuevo aporte en relación con la interdigitación (sensu Martínez Cereceda, 1999) de las poblaciones del Formativo, que se complementa con el registro de evidente interacción con San Pedro de Atacama en contextos contemporáneos (Otero y Rivolta, 2015; Tarragó, 1977).

Para finalizar, concluimos que, si bien los materiales procedentes de tareas de salvataje son escasamente atendidos en nuestro país, en Tilcara la Arqueología de Rescate representó, en principio, la única oportunidad de aproximarnos al registro para conocer las características de las aldeas más antiguas asentadas en el sector central de la Quebrada de Humahuaca. El abordaje de estos contextos, que en ocasiones se ve condicionado por el tiempo en el que se pueden desarrollar las excavaciones (por la necesidad, por ejemplo, de continuar con las obras viales u otras construcciones) y la recomposición de los contextos de hallazgo a partir de la documentación elaborada por otros investigadores, representa un reto a superar con el uso de diferentes estrategias analíticas, como las aquí planteadas desde el estudio de la alfarería. El ejido de Tilcara, por su dinámica natural y constante modificación por la acción antrópica lleva a la exposición de restos arqueológicos de manera continua. En este sentido, la arqueología de rescate se presenta como una oportunidad para profundizar sobre las primeras ocupaciones de este sector de la Quebrada, en el que la cerámica se mantiene como una de las principales fuentes de información.

San Salvador de Jujuy, 14 de mayo de 2020.

\section{Bibliografía}

Balfet, H., Fauvet-Berthelot, M. F., y Monzón, S. (1983). Pour la Normalisation de la Description des Poteries. Paris, France: Editions du Centre National de la Recherche Scientifique.

Carlos Ríos, E. (2015). La circulación entre mundos en la tradición oral y ritual y las categorías del pensamiento quechua: el Hanansaya ccullana Chisikata (Cusco, Perú). (Tesis doctoral inédita). Universitat Autónoma de Barcelona, España.

Courtois, L. (1976). Examen au Microscope Petrographique des Ceramiques Archeologiques. Notes et Monographies Techniques 8. Paris, Francia: Centre National de la Recherche Scientifique.

Cremonte, M. B. (2006). El estudio de la cerámica en la reconstrucción de las historias locales. El sur de la Quebrada de Humahuaca (Jujuy, Argentina) durante los Desarrollos Regionales e Incaico. Chungara Revista de Antropología Chilena 38, 239-247.

Cremonte, M. B. y Bugliani, M. F. (2006- 2009). Pasta, forma e iconografía. Estrategias para el estudio de la cerámica arqueológica. Xama 19- 23, 239-262.

González, M. A., Tchilinguarian, P., Pereyra, F. X., Ramallo, E. E., y González, O. E. (2003). Hoja Geológica 2366-IV, Ciudad de Libertador General San Martín. Provincias de Jujuy y Salta. Instituto de Geología y Recursos Minerales, Servicio Geológico Minero Argentino. Boletín 274, SEGEMAR, Buenos Aires.

Larcher, N. E., Otero, C., Cremonte, M. B. (2016). Caracterización y procedencia de materias primas para la producción de alfarería prehispánica en la Quebrada de Humahuaca (Provincia de Jujuy, República Argentina). FRX, ICP y petrografía de pastas. Arqueología Iberoamericana 29, 50-56.

Madrazo, G. (1968). Alfarería prehumahuaca en Tilcara (Quebrada de Humahuaca, Prov. de Jujuy). Etnía 8, 16-18.

Madrazo, G. (1969). Reapertura de la investigación en Alfarcito (Provincia de Jujuy, República Argentina). Monografías del Museo Etnográfico Municipal "Dámaso Arce" 4, 1-70.

Martínez Cereceda, J. L. (1999). Ayllus e identidades interdigitadas. Las sociedades de la Puna Salada. En Boccara, G. y Galindo, S. (Eds.), Lógica mestiza en América (pp. 85-112). Temuco: Instituto de Estudios Indígenas.

Menacho, K. A. (2007). Etnoarqueología y estudios sobre 
funcionalidad cerámica: aportes a partir de un caso de estudio. Intersecciones en Antropología 8, 149-161.

Menacho, K. A. y González, N. (2005). La alfarería de "Malka": Formativo Final de la Quebrada de Humahuaca. Cuadernos FHyCSUNJU 26, 186-187.

Mendonça, O. J., Bordach, M. A., Ruiz, M. S., y Cremonte, M. B. (1991). Nuevas evidencias del Periodo Agroalfarero Temprano en Quebrada de Humahuaca. Los hallazgos del Sitio Til. 20 (Tilcara, Jujuy). Comechingonia. Revista de Antropología e Historia 8 (7), 29-48.

Nielsen. A. (2001). Evolución social en la Quebrada de Humahuaca (AD 700-1536). En Berberián, E. y Nielsen, A. (Ed.), Historia Argentina Prehispánica. Tomo I (pp. 171-264). Córdoba, Argentina: Editorial Brujas.

Nielsen, A. (2007). El Período de Desarrollos Regionales en la quebrada de Humahuaca: aspectos cronológicos. En Williams, V., Ventura, B., Callegari, A. y Yacobaccio, H. (Eds.), Sociedades precolombinas surandinas. Temporalidad, interacción y dinámica cultural del NOA en el ámbito de los Andes Centro-Sur (pp. 235-251). Buenos Aires: Taller Internacional del NOA y Andes Centro Sur.

Núñez, L., Cartajena, I., Carrasco, C., De Souza, P., y Grosjean, M. (2006). Emergencia de comunidades pastoralistas formativas en el sureste de la Puna de Atacama. Estudios Atacameños. Arqueología y Antropología Surandinas 32, 93-117.

Olivera, D. E. y Palma, J. R. (1997). Cronología y registro arqueológico en el Formativo Temprano en la región de Huma huaca. Avances en Arqueología 3, 77-99.

Ortiz, M. G. y Delgado, A. (1997). La Cerámica del Pucara de Volcán. Jujuy, Argentina: EDIUNJu.

Orton, C., Tyres, P., Vince, A. (1997). La cerámica en Arqueología. Barcelona, España: Editorial Crítica.

Otero, C. (2013). Producción, usos y circulación de bienes en el Pucará de Tilcara (Quebrada de Humahuaca, Jujuy). Tesis doctoral inédita. Facultad de Filosofía y Letras, Universidad de Buenos Aires, Buenos Aires.

Otero, C. (2017). Ceramics styles from Pucara de Tilcara settlement during inca domination. En Scaro, A., Otero, C. y Cremonte, M. B. (Ed.), Pre-Inca and Inca Pottery. Quebrada de Humahuaca, Argentina (pp. 107-138). Cham: Springer.

Otero C. y Cremonte, M. B. 2010. Los objetos cerámicos en la dinámica social de la Unidad 1 del Pukara de Tilcara (Quebrada de Humahuaca, Jujuy). Actas del XVII Congreso
Nacional de Arqueología Argentina, Tomo I, 181-186. Mendoza, Argentina.

Otero, C. y Mercolli, P. (2008). Consumo y circulación de objetos cerámicos de Tilcara. Tres ejemplos de distintos contextos funerarios. IX Jornadas Regionales de Investigación en Humanidades y Ciencias Sociales. Suplemento de Cuadernos 34, 227- 228. Jujuy, Argentina.

Otero, C. y Rivolta.M. C. (2015). Nuevas interpretaciones para la secuencia de ocupación de Tilcara (Quebrada de Humahuaca, Jujuy). Intersecciones en Antropología 16, 145-159.

Pereyra Domingorena, L. y Cremonte, M. B. (2017). The Same Way of Doing Pottery. San Francisco Ceramic Fabrics from Tumbaya (Quebrada de Humahuaca) and San Pedro (San Francisco River Basin). En Scaro, A., Otero, C. y Cremonte, M. B. (Ed.), Pre-Inca and Inca Pottery. Quebrada de Humahuaca, Argentina (pp. 1-27). Cham: Springer.

Pimentel, G. (2009). Las huacas del tráfico. Arquitectura ceremonial en rutas prehispánicas del Desierto de Atacama. Boletín del Museo Chileno de Arte Precolombino 14:9-38.

Rice, P. (1987). Pottery Analysis. Chicago, USA: University of Chicago Press.

Rivolta, M. C. (1996). Calle Lavalle y Sorpresa: Aportes a la Investigación Arqueológica de la Quebrada de Humahuaca. XXV Aniversario Museo Arqueológico Dr. Eduardo Casanova, 129-135. Tilcara, Jujuy: Instituto Interdisciplinario Tilcara.

Rivolta, M. C. y Albeck, M. E. (1992). Los asentamientos tempranos en la Localidad de Tilcara: S.Juj. Til.22, Provincia de Jujuy. Cuadernos 3, 86-93.

Rivolta, M.C. y Otero, C. (2016). El Estilo como articulador: cambios y continuidades en la cerámica de las sociedades Formativas. Actas del XIX Congreso Nacional de Arqueología Argentina, Serie Monográfica y Didáctica 54, 1807-1812. San Miguel de Tucumán, Argentina.

Rivolta, M.C. y Otero, C., y Greco, C. (2017). Reconsidering Isla Occupation. Pottery, Chronology and Settlement. En. Scaro, A., Otero, C. y Cremonte, M. B. (Eds.), Pre-Inca and Inca pottery, Quebrada de Humahuaca, Argentina (pp.29-50). Cham: Springer.

Rivolta, M. C., Seldes, V. y Mercolli, P. (2010). Ocupaciones Tempranas en sectores Urbanos de la localidad de Tilcara (Jujuy, Argentina). Actas del XVII Congreso Nacional de Arqueología Chilena. Vol 1, 155-163). Valdivia, Chile.

Rye, O. S. (1981). Pottery Technology. Principles and 
reconstrunction. Manuals on archaeology, 4. Washington D. C: Taraxacum.

Skibo, J. M. (1992). Pottery Function. A Use-Alteration Perspecive. New York, EEUU: Plenum Press.

Tarragó, M. N. 1977 Relaciones Prehispánicas entre San Pedro de Atacama (norte de Chile) y regiones aledañas: La Quebrada de Humahuaca. Estudios Atacameños 5, 50-63.

Tarragó, M. y Albeck, M.E. (1997). Fechados radiocarbónicos para el sector medio de la Quebrada. Avances en Arqueología 3, 101-129.
Uribe, M., 2002. Sobre alfarería, cementerios, fases, procesos y la construcción de Atacama en la prehistoria Tardía (800-1600 DC). Estudios Atacameños 22: 7-31.

Valda, P. A. (2010). Informe final correspondiente a los trabajos de rescate realizados en el barrio Malka, Localidad de Tilcara, Jujuy. (Informe inédito). Instituto Interdisciplinario Tilcara, Facultad de Filosofía y Letras. Universidad de Buenos Aires.

Zaburlin, M. A. (2009). Ocupación del Formativo Final en la Puna jujeña el caso de Santa Ana de Abralaite. Trabajo presentado en el VI Encuentro Interdisciplinario de Ciencias Sociales y Humanas, Córdoba, Argentina. 